\title{
Un modelo para la autoevaluación de la calidad de programas de educación universitaria virtual
}

\section{Model for the self-assessment of the quality of higher education online programmes}

\author{
Renata Marciniak \\ Universidad Autònoma de Barcelona, Barcelona, España \\ marciniak.renata@e-campus.uab.cat \\ Joaquín Gairín Sallán \\ Universitat Autònoma de Barcelona, Barcelona, España \\ joaquin.gairin@uab.es
}

\begin{abstract}
Resumen
La mejora de la calidad de programas formativos impartidos bajo la modalidad virtual es un gran desafío para todas las universidades que los ofrecen, si consideramos las crecientes expectativas de los estudiantes y los requisitos de calidad y mejora permanente de las agencias de acreditación. Uno de los métodos que permiten identificar el nivel actual de la calidad del programa y mejorarlo es la autoevaluación que debería abarcar la evaluación de la calidad propia del programa, así como su evaluación continua. El objetivo del artículo es presentar un modelo aplicativo para la autoevaluación de programas de educación universitaria virtual, que integra los dos tipos de evaluación y de esta manera permite evaluar la calidad tanto de los elementos pedagógicos y tecnológicos que constituyen un programa virtual como cada una de las tres fases de su aplicación: la fase inicial, de desarrollo y la fase final. La validación del modelo ha incluido varios momentos e implicado a 23 expertos internacionales y su aplicación en la autoevaluación de cuatro programas virtuales. El modelo está dirigido a los responsables de la ejecución de programas formativos impartidos bajo la modalidad virtual, directores/coordinadores de las carreras a las que pertenece el programa. Asimismo, puede servir a los diseñadores de programas virtuales como pauta para diseñar un programa virtual de buena calidad.
\end{abstract}

Palabras clave: autoevaluación, calidad, educación virtual, modelo, programa formativo.

\begin{abstract}
The improvement of the quality of online education programmes is a challenge for all universities offering the said programmes. Due to students' and accreditation agencies' growing expectations, the universities are required to develop a focus towards the quality of virtual education programmes and application of methods in order to help the improvement of the said quality. Among other methods, we encounter the self-assessment of the programme, which should consider the assessment of the quality of the program itself, as well as, its on-going assessment. One of the methods that allows to determine the current level of the quality of the programme and to improve it is the self-assessment, that should include the assessment of the quality of the programme itself, as well as its continuous assessment. The aim of this paper is to present an applicative model for the self-
\end{abstract}


assessment of the higher education virtual programmes that includes both types of assessment and, thus, allow to assess all pedagogical and technological elements of the programme, as well as the three stages the program goes through (initial, processual and final). The validation of the model included several moments and involved 23 international experts in online education, as well as the application of the model in the self-assessment of four programmes. The model provides guidance for the persons in charge of the online programme in order for them to carry out a more comprehensive assessment of its quality. It can also be used by the designers of online programmes and by supervisors of the quality of higher education.

Keywords: self-assessment, quality, online education, model, education programme.

\section{Introducción}

La calidad de los programas de educación universitaria virtual es objeto de interés de muchas universidades que los ofrecen. Su alta calidad y su evaluación continua condicionan el desarrollo de la educación virtual que, en la era de difusión de la educación abierta y a distancia, se convierte en un deber de la formación universitaria.

La calidad de los programas de educación universitaria virtual es un aspecto complejo, sobre todo, por su carácter multifactorial. Algunos autores relacionan dicha calidad con la "satisfacción de usuarios, excelencia del sistema, efectividad del proceso, resultados académicos positivos y buen impacto social del programa" (Fainholc, 2004, p. 2). Para Ruiz y Dávila (2016), "la calidad de la formación en la modalidad virtual es una función de las interacciones complejas que se producen entre los diferentes factores que intervienen en los resultados de aprendizaje para satisfacer necesidades personales y sociales" (p. 17). En la opinión de García Aretio (2014) "cuando hablamos de calidad asumimos que nos queremos referir a la buena calidad, o a la excelencia, o a la eficacia en grado sumo. Así, si hablamos de buena calidad de educación a distancia nos deberíamos estar refiriendo a una educación a distancia eficaz y de calidad (positiva)" (p. 115-116). Nosotros entendemos la calidad de los antemencionados programas como el proceso de esforzarse por conseguir ciertas dimensiones que conforman la calidad de dichos programas e indicadores asociados a ellas.

Para garantizar la calidad de los mencionados programas, uno de los estándares definidos por la European Association for Quality Assurance in Higher Education, adoptados por los ministros de educación europeos en Bergen en 2005, establece que las universidades deberían disponer de mecanismos formales para la aprobación, revisión periódica y control de sus programas (tanto presenciales como virtuales) y titulaciones (ENQA, 2015). La realización de este compromiso hace realidad la autoevaluación de programas, que se puede definir como:

"Un proceso sistemático orientado al aseguramiento y mejora de programas, realizado por el Comité de Autoevaluación compuesto por las personas involucradas en la ejecución del programa (y otras nombradas por la universidad si conviene). Dicho Comité evalúa el nivel de calidad del programa a partir de un conjunto de indicadores de calidad, utilizando diferentes fuentes de información. Los resultados de esta

Un modelo para la autoevaluación de la calidad de programas de educación universitaria virtual. Renata Marciniak y Joaquín Gairín Sallán.

Página 2 de 30 
evaluación se utilizan para elaborar el plan de mejora de los áreas del programa que presentan un bajo nivel de calidad" (Marciniak, 2016, p. 157).

Algunos investigadores (Rausaria y Lele, 2002; Sarramona, 2001; Grifoll et al., 2010) señalan que la autoevaluación debería ser una práctica permanente que posibilite una mirada crítica a las personas responsables de la ejecución del programa virtual y a la forma de como asumen el desarrollo de todos los aspectos que influyen en la calidad del programa y aseguran el alto nivel de ésta.

Para que la autoevaluación sea una herramienta útil a la hora de revisar programas de educación virtual e implementar los cambios o acciones de su mejora detectadas a través de la misma, debe ser realizada según un modelo que tenga en cuenta el contexto específico de la modalidad virtual de educación (Marciniak, 2016). Cabe entender que modelos de otras áreas pueden no resultar pertinentes, dado que desatienden aspectos relevantes en la educación virtual como, por ejemplo, estrategias didácticas específicas, materiales didácticos digitales, tutoría o "las posibilidades y limitaciones en la interacción entre profesor y alumno o entre éstos últimos" (Bautista, Martínez y Sainz, 2001, p. 6).

Actualmente, existen numerosos modelos desarrollados para evaluar la educación virtual como los mencionados por Domínguez y Morcillo (2016), Hilera (2010), Motz (2013), Quesada et al. (2015), Marciniak y Gairín (2017), Rodríguez y Torres (2016). Cada uno de ellos tiene como objetivo ayudar a las universidades a mejorar la calidad de dicha educación. Sin embargo, además de sus ventajas, presentan ciertas desventajas. Una de ellas es que los indicadores que se proponen en estos modelos raramente avalan la necesidad de evaluar tanto la calidad del propio programa, como su planificación, aplicación e impacto, tal como postulan algunos autores y entre ellos Martínez (2013) cuando sostiene que:

"La evaluación de programas debe recoger la información sistemática acerca de un programa elaborado para atender a unas necesidades, centrada en 1) la calidad del propio programa, en sus fundamentos, estructura y coherencia; 2) la planificación de su puesta en acción, considerando los recursos personales, materiales y organizativos; 3) el programa en su desarrollo, en la acción, y 4) sus resultados, en el inmediato, medio y largo plazo, con el objetivo de comprobar y valorar el grado y calidad con el que ha conseguido atender las necesidades y resolver los problemas que eran la razón del ser del programa (Martínez, 2013, p. 197).

El objetivo de este artículo ${ }^{1}$ es mostrar los resultados obtenidos en el diseño de un modelo aplicativo para la autoevaluación de programas de educación universitaria virtual, que integre la evaluación de la calidad de propio programa virtual y la evaluación continua del mismo. De esta manera, se espera que el modelo se convierta en una herramienta útil para evaluar y mejorar todos los elementos que constituyen un programa impartido bajo la modalidad virtual y cada una de las tres fases por las que el programa pasa durante su existencia; es decir, la fase inicial, la fase de desarrollo y la fase final. Cabría insistir que este enfoque global no ha sido abordado (o lo ha sido parcialmente) como indican autores como Butcher y Wilson-Strydom (2013), Chmielewski (2013), Marciniak (2016).

\footnotetext{
${ }^{1}$ En este artículo se presenta de una forma panorámica el proceso y - particularmente - los hallazgos de la investigación propia de una tesis doctoral realizada en la UAB entre los años 2013-2016; tesis que versa sobre la Autoevaluación de programas de educación universitaria virtual.

Un modelo para la autoevaluación de la calidad de programas de educación universitaria virtual. Renata Marciniak y Joaquín Gairín Sallán. 


\section{Programa formativo virtual y sus elementos}

Entendemos un programa virtual como:

"Un documento (informatizado o no) que identifica acciones orientadas a lograr objetivos formativos para un periodo determinado, y que incluye un conjunto de elementos necesarios para el desarrollo del proceso de enseñanza-aprendizaje de una asignatura determinada impartida en la modalidad virtual. Se realiza completamente mediante a las Tecnologías de la Información y Comunicación, con el soporte tecnológico de una plataforma educativa virtual y con apoyo de un/os docente/s en línea, quien/es orienta/n el desarrollo académico de los estudiantes utilizando diversas vías de comunicación sincrónica y asincrónica" (Marciniak, 2016, p. 70).

En cuanto a los elementos del programa formativo virtual debe señalarse que no existen fórmulas o esquemas para la elaboración y el diseño de un programa virtual; cada programa es único, y su estructura y funcionamiento dependerá de sus objetivos, destinatarios, contenidos, recursos, etc. Sin embargo, en el documento "Directrices para la Educación a Distancia" elaborados por el Higher Education Committee on Quality (HECQ, 1997) se han definido algunos estándares académicos de los programas de educación superior no presencial y qué elementos deben incluirse en ellos para que los programas sean considerados de buena calidad. Con respecto a lo segundo cabe citar lo que el HECQ, lo indicado en el precepto 7 y 8 de dicho documento:

\section{"Precepto 7:}

En el diseño de programas de estudio a distancia y en cualquier módulo del programa la institución proveedora debe asegurar coherencia explícita y razonable entre, por un lado, las metas y los resultados del aprendizaje, y, por otro las estrategias de enseñanza a distancia, la competencia de los materiales de aprendizaje y los modos y criterios de valoración.

Precepto 8:

"Una institución proveedora es responsable de asegurar que el diseño y el desarrollo de los programas de aprendizaje a distancia sean de alta calidad y proporcionen al estudiante una oportunidad justa y razonable de alcanzar los estándares académicos necesarios para llegar con éxito al final".

Por ello, se espera que la universidad:

- Tome en cuenta las habilidades, el conocimiento y la experiencia de los estudiantes matriculados y de las circunstancias en las cuales se espera que éstos hagan uso del sistema de distancia de la institución.

- Explicite los principios básicos para seleccionar los medios de enseñanza elegidos.

- Considere y especifique de forma precisa y detallada los recursos de aprendizaje y ayuda que serán puestos a disposición directa de los estudiantes por la institución proveedora.

- Considere cómo conseguir que los materiales de aprendizaje sean interactivos y permitir la retroalimentación formativa de los estudiantes.

- Especifique detalladamente el horario de las actividades del estudiante en relación con el programa de estudio usando el sistema previsto para el aprendizaje a distancia" (HECQ, 1997, p.9-10).

Un modelo para la autoevaluación de la calidad de programas de educación universitaria virtual. Renata Marciniak y Joaquín Gairín Sallán. 
Tomando como base las precitadas directrices, puede afirmarse que un programa virtual debe incluir los siguientes elementos:

- metas de aprendizaje,

- competencias,

- estrategias de enseñanza,

- estrategias de evaluación del aprendizaje,

- perfil del estudiante,

- materiales educativos interactivos,

- recursos de aprendizaje,

- recursos del apoyo a los estudiantes,

- contenido temático,

- cronograma de actividades del estudiante.

La tabla 1 recoge los elementos que, según otros autores, se deberían tener en cuenta a la hora de diseñar un programa formativo virtual.

\begin{tabular}{|c|c|}
\hline Autor & Elementos del programa virtual \\
\hline Meza (2012) & $\begin{array}{l}\text { - Aspectos específicos del programa } \\
\text { - Temas y objetivos } \\
\text { - Actividades de aprendizaje } \\
\text { - Evaluación del aprendizaje } \\
\text { - Tutoría virtual }\end{array}$ \\
\hline $\begin{array}{l}\text { Mery y Newby } \\
(2014)\end{array}$ & $\begin{array}{l}\text { - } \text { Necesidades de los estudiantes } \\
\text { - Objetivos formativos } \\
\text { - Competencias } \\
\text { - Contenidos } \\
\text { - Material didáctico } \\
\text { - Actividades de aprendizaje } \\
\text { - Evaluación del aprendizaje } \\
\text { - Evaluación del programa }\end{array}$ \\
\hline Shattuck (2014) & $\begin{array}{l}\text { - Aspectos específicos del programa (nombre, duración, justificación) } \\
\text { - Objetivos y contenidos } \\
\text { - Materiales multimedia e interactivos } \\
\text { - Actividades de aprendizaje } \\
\text { - Evaluación del aprendizaje } \\
\text { - Tutorías } \\
\end{array}$ \\
\hline $\begin{array}{c}\text { Barnard y } \\
\text { Echols (2015) }\end{array}$ & $\begin{array}{l}\text { - } \text { Perfil del estudiante } \\
\text { - } \text { Contenidos temáticos } \\
\text { - Estrategias de aprendizaje } \\
\text { - Características de tecnología educativa utilizada } \\
\text { - } \quad \text { Evaluación del aprendizaje } \\
\end{array}$ \\
\hline $\begin{array}{l}\text { Zapata } \\
(2015)\end{array}$ & $\begin{array}{l}\text { - Objetivos y epítome } \\
\text { - Unidades } \\
\text { - Guías docentes y guías didácticas de las unidades } \\
\text { - Materiales para cada unidad } \\
\text { - Tipos y funciones de los profesores } \\
\end{array}$ \\
\hline $\begin{array}{c}\text { Boettcher y } \\
\text { Conrad (2016) }\end{array}$ & $\begin{array}{l}\text { - Objetivos y metas a alcanzar, } \\
\text { - Recursos didácticos. } \\
\text { - Acciones de aprendizaje. } \\
\text { - Estrategias de evaluación de aprendizaje. }\end{array}$ \\
\hline $\begin{array}{l}\text { Rushby y Surry } \\
\text { (2016) }\end{array}$ & $\begin{array}{l}\text { - Datos curriculares } \\
\text { - Objetivos generales de aprendizaje } \\
\text { - Contenidos del programa }\end{array}$ \\
\hline
\end{tabular}

Un modelo para la autoevaluación de la calidad de programas de educación universitaria virtual. Renata Marciniak y Joaquín Gairín Sallán.

Página 5 de 30 


\begin{tabular}{|l|l|}
\hline & $\bullet$ Actividades del aprendizaje \\
& $\bullet$ Fuentes de información \\
& $\bullet$ Evaluación del aprendizaje \\
\hline
\end{tabular}

Tabla 1. Propuestas de elementos del programa formativo virtual según diferentes autores

Al analizar las directrices de HECQ (1997) y propuestas de algunos autores (Tabla 1), podemos notar que nos ofrecen indicaciones sobre los elementos del programa formativo virtual que debe abarcar el programa virtual. Los más comunes son:

- Objetivos y competencias de aprendizaje;

- Estrategias de enseñanza;

- Contenido temático;

- Materiales y recursos didácticos;

- Actividades de aprendizaje;

- Estrategias de evaluación del aprendizaje.

\section{Modelos de referencia de autoevaluación de programas formativos virtuales}

Actualmente, existen muchos modelos que pueden ser utilizados para evaluar un programa de educación virtual, sin embargo no todos ellos presentan un enfoque coincidente (Sarramona, 2001; Fidalgo, 2007; Marshall, 2007; Marcelo y Zapata, 2008; Díaz-Maroto, 2009; University of Wisconsin, UW, 2008; Asociación de E-learning Académico de Polonia, SEA, 2010; Instituto Latinoamericano y del Caribe de Calidad en Educación Superior a Distancia, CALED, 2010; Online Learning Consortium, OLC, 2011; Zhang y Jiang, 2012; Giorgetti et al., 2013; Africa Virtual University, AVU, 2014; European Association of Distance Teaching Universities, EADTU, 2016, entre otros). Por ejemplo, Fidalgo (2007) señala la existencia de cinco factores importantes en la evaluación de la calidad del programa online: 1) Contenidos de aprendizaje, en cuanto al conjunto de recursos que se utiliza para el aprendizaje; 2) Organización de contenidos, en relación con la forma en que los participantes del programa ven la estructura del mismo; 3) Retroalimentación del alumnado, referida a acreditar que el usuario ha realizado unas determinadas acciones por su parte; 4) Acción tutorial, en cuanto a la atención del tutor al alumno para resolver dudas, realizar seguimiento y funciones de animación, y 5) Otros indicadores, tales como curva de agotamiento, factor de humanización o relación de contenidos.

El E-learning Maturity Model (eMM) desarrollado por Marshall (2007) propone evaluar los siguientes aspectos de programa en línea: 1) Aprendizaje (los aspectos pedagógicos del proceso educativo); 2) Desarrollo (el proceso de creación y mantenimiento de los recursos de e-learning); 3) Ayuda ( el proceso de apoyo y gestión operativos del curso); 4) Evaluación ( evaluación de aprendizaje y control de calidad de e-learning en todo su ciclo de vida), y 5) Organización (el proceso relacionado con el planeamiento institucional y la gestión organizativa).

Un modelo para la autoevaluación de la calidad de programas de educación universitaria virtual. Renata Marciniak y Joaquín Gairín Sallán. 
A su vez, Marcelo y Zapata (2008) consideran que la evaluación de un programa virtual debería abarcar la evaluación del contexto para ver si el programa se adecúa a las necesidades, características y condiciones del mismo; el diseño de programa (objetivos, contenidos, estrategias y recursos), la producción del programa (requerimientos técnicos de la plataforma tecnológica, su usabilidad y accesibilidad), la puesta en marcha de programa (procesos de difusión, información y orientación al profesorado respecto de las características y condiciones del programa), la implementación del programa (competencias de los tutores, proceso formativo, desarrollo de una evaluación inicial, formativa final del programa), y el seguimiento de programa (promoción de asesoramiento continuado, espacios virtuales de comunicación e intercambio entre los participantes del programa).

Las dimensiones propuestas por el modelo de evaluación de e-learning académico diseñado por la Asociación de E-learning Académico de Polonia (SEA, 2010), sirven de ayuda a las universidades para llevar a cabo la autoevaluación de programas virtuales tanto los ya existentes como los que se están diseñando. Dichas dimensiones son: 1) Organización del programa (abarca criterios para evaluar si se han creado condiciones para realizar el programa correctamente y con eficacia); 2) Diseño del programa (incluye criterios que se refieren, por un lado, a la metodología del programa, y, por otro, a su calidad en el aspecto tecnológico); 3) Desarrollo del programa (abarca criterios para evaluar la realización del programa en el contexto organizativo determinado) y 4) Evaluación del programa (se valora si el proceso de evaluación del programa ha sido correctamente planteado y realizado).

Los modelos mencionados anteriormente no son los únicos desarrollados para evaluar la calidad de la educación virtual, ya que existen otros tantos. La variedad de estos modelos se debe a "la necesidad de contar con un marco propio de referencia para e-learning" (Zapata, 2006, p. 1). Sin embargo, la mayoría de dichos modelos aborda desde diferente perspectiva la evaluación del programa formativo impartido en la modalidad virtual. La Tabla 2 presenta elementos y fases de programa que evalúan los modelos analizados durante la investigación bibliográfica. La presentación se hace cronológicamente. 


\begin{tabular}{|c|c|c|c|c|c|c|c|c|c|c|c|c|c|c|c|}
\hline $\begin{array}{l}\text { Modelo } \\
\text { Elemento de Programa }\end{array}$ & 1 & 2 & 3 & 4 & 5 & 6 & 7 & 8 & 9 & 10 & 11 & 12 & 13 & 14 & 15 \\
\hline Justificación del programa & & $\mathrm{X}$ & & & & & & & & & & & & & \\
\hline Objetivos del programa & $\mathrm{x}$ & $\mathrm{x}$ & & $\mathrm{X}$ & $\mathrm{x}$ & & $\mathrm{x}$ & & $\mathrm{x}$ & & & $\mathrm{x}$ & & $\mathrm{x}$ & \\
\hline Perfil del estudiante & $\mathrm{x}$ & $\mathrm{X}$ & & $\mathrm{X}$ & $\mathrm{X}$ & & & & $\mathrm{x}$ & & $\mathrm{x}$ & $\mathrm{x}$ & & $\mathrm{x}$ & $\mathrm{x}$ \\
\hline Contenidos temáticos & & $\mathrm{X}$ & X & X & X & $\mathrm{x}$ & $\mathrm{x}$ & & $\mathrm{x}$ & $\mathrm{x}$ & $\mathrm{x}$ & & & & \\
\hline Actividades de aprendizaje & & $\mathrm{x}$ & & & $\mathrm{x}$ & & $\mathrm{x}$ & $\mathrm{x}$ & $\mathrm{x}$ & $\mathrm{x}$ & $\mathrm{x}$ & $\mathrm{x}$ & $\mathrm{x}$ & & $\mathrm{x}$ \\
\hline $\begin{array}{l}\text { Perfil de los docentes en } \\
\text { línea }\end{array}$ & $\mathrm{x}$ & & & $\mathrm{X}$ & $\mathrm{X}$ & & $\mathrm{x}$ & & $\mathrm{x}$ & & $\mathrm{x}$ & $\mathrm{x}$ & & $\mathrm{x}$ & \\
\hline Tutorías & & & $\mathrm{x}$ & & & & $\mathrm{x}$ & & $\mathrm{x}$ & & & $\mathrm{X}$ & & & \\
\hline $\begin{array}{l}\text { Materiales y recursos } \\
\text { didácticos }\end{array}$ & & $\mathrm{x}$ & $\mathrm{x}$ & & $\mathrm{x}$ & $\mathrm{x}$ & $\mathrm{x}$ & $\mathrm{x}$ & $\mathrm{x}$ & $\mathrm{x}$ & $\mathrm{x}$ & $\mathrm{X}$ & $\mathrm{X}$ & $\mathrm{X}$ & $\mathrm{x}$ \\
\hline $\begin{array}{l}\text { Metodologías de enseñanza } \\
\text { y aprendizaje }\end{array}$ & & $\mathrm{X}$ & & $\mathrm{X}$ & X & & $\mathrm{x}$ & & $\mathrm{x}$ & $\mathrm{x}$ & $\mathrm{x}$ & & & $\mathrm{x}$ & \\
\hline Evaluación del aprendizaje & & $\mathrm{x}$ & $\mathrm{x}$ & & $\mathrm{x}$ & $\mathrm{x}$ & $\mathrm{x}$ & $\mathrm{x}$ & $\mathrm{x}$ & & $x$ & $\mathrm{x}$ & $\mathrm{x}$ & $\mathrm{x}$ & \\
\hline Plataforma virtual & $\mathrm{x}$ & & & $\mathrm{x}$ & $\mathrm{x}$ & & $\mathrm{x}$ & & & $\mathrm{X}$ & $\mathrm{x}$ & $\mathrm{X}$ & & $\mathrm{x}$ & \\
\hline $\begin{array}{l}\text { Evaluación de la fase inicial } \\
\text { del programa }\end{array}$ & & $\mathrm{X}$ & $\mathrm{x}$ & $\mathrm{x}$ & $\mathrm{x}$ & & & $\mathrm{x}$ & & & & & & X & $\mathrm{X}$ \\
\hline $\begin{array}{l}\text { Evaluación de la fase de } \\
\text { desarrollo del programa }\end{array}$ & & & & $\mathrm{x}$ & $\mathrm{x}$ & & & & & $\mathrm{X}$ & & & & $\mathrm{X}$ & $\mathrm{x}$ \\
\hline $\begin{array}{l}\text { Evaluación de la fase final } \\
\text { del programa }\end{array}$ & & $\mathrm{x}$ & & $\mathrm{x}$ & $\mathrm{x}$ & $\mathrm{x}$ & & $\mathrm{x}$ & & $\mathrm{x}$ & $\mathrm{X}$ & $\mathrm{x}$ & & $\mathrm{X}$ & \\
\hline
\end{tabular}

Explicación: 1- Sarramona, 2001; 2-Fidalgo, 2007; 3-Marshall, 2007; 4-Marcelo y Zapata, 2008; 5-Ejarque et al. 2008; 6-UW, 2008; 7-Díaz-Maroto, 2009; 8-SEA, 2010; 9-CALED, 2010; 10-OLC; 2011; 11- Masoumi y Lindström, 2012; 12-Zhang y Jiang, 2012; 13-Giorgetti et al., 2013; 14-AVU, 2014; 15-EADTU, 2016.

Tabla 2. Cuadro comparativo. Elementos de programa que evalúan los modelos analizados

Los diferentes modelos (Tabla 2) intentan dar respuesta al tema de la evaluación de programas de educación universitaria virtual. Sin embargo, hasta el momento ninguno logra cubrir por si solo todas las necesidades evaluativas de estos programas desde el punto de vista de la calidad. Se hace preciso identificar dimensiones e indicadores que permitan medir la calidad de todos los elementos pedagógicos y tecnológicos del programa en cada una de las tres fases de su existencia. Si éstos no se evalúan, no se cuenta con la información y el marco de posibles decisiones necesarios para mejorar los elementos y las fases del programa que presentan un bajo nivel de calidad. Para llenar este hueco, proponemos un modelo alternativo - el modelo de autoevaluación de programas de educación universitaria virtual - que permite a las universidades evaluar tanto la calidad de todos los elementos que constituyen el programa (evaluación de la calidad del propio programa), como la calidad de su planificación, desarrollo y resultados (evaluación continua del programa).

\section{Metodología}

La base del diseño del Modelo de Autoevaluación de Programas de Educación Universitaria Virtual fue un detallado análisis de las diferentes propuestas de elementos de programas formativos realizadas por distintos autores (ver Tabla 1), así como del Un modelo para la autoevaluación de la calidad de programas de educación universitaria virtual. Renata Marciniak y Joaquín Gairín Sallán. 
estudio documental sobre estándares, normas, directrices, modelos y guías ya existentes para llevar a cabo la evaluación de educación virtual (Abdulaziz, Almetair y Alzeer, 2014; ADL, 2014; AENOR, 2012; AICCJC, 2013; Attwell, 2006; AVU, 2014; CALED, 2010; CEN, 2007; CIEES, 2008; EADTU, 2016; EFQUEL, 2010; Ehlers, 2013; García Aretio, 1998; Hilera y Hoya, 2010; IEEE, 2014; ISO/IEC 19796-1:2005; Kirkpatrick, 2007; Latchem, 2014; Marshall, 2007; Masoumi y Lindström, 2012; MSCHE, 2011; Marciniak, 2015; Nesbit, Belfer, y Vargo, 2002; UNIQUe, 2014; Zhang y Jiang, 2012; entre otros). A partir de dicho análisis pudimos extraer las variables y dimensiones que se deben tener en cuenta a la hora de evaluar un programa formativo impartido bajo la modalidad virtual y diseñar el primer borrador del modelo (modelo provisional I) compuesto por dos variables, catorce dimensiones y ciento veinte indicadores.

El modelo provisional I se validó por 23 expertos de diferentes países (España, Portugal, Italia, México, Chile, Paraguay, Uruguay), seleccionados por tener experiencias en el campo y por haber escrito sobre el mismo. Para seleccionar los expertos mediante correo electrónico, contactamos con un total de 42 personas vinculadas con el diseño, desarrollo o evaluación de programas universitarios y programas virtuales, diseño o evaluación de materiales y recursos didácticos destinados a la educación virtual y con personas relacionadas con el diseño, administración o evaluación de aulas virtuales solicitando su colaboración en el proceso de validación del modelo provisional I. Fueron 23 personas las que aceptaron nuestra invitación, entre ellas, el Director de una Agencia Española de Evaluación de Calidad de la Educación Superior, 4 profesores universitarios que enseñan en línea, 5 autores de publicaciones relacionados con la evaluación de la calidad de educación virtual, 3 asesores metodológicos de programas virtuales, 4 expertos que se dedican a la elaboración y evaluación de la calidad de materiales y recursos didácticos digitales, así como a 3 expertos que se dedican a la evaluación del aula virtual y los 3 administradores de las mismas. Se pidió a los expertos que determinasen la validez de cada una de las dimensiones y los indicadores que conforman el modelo, así como la apropiación de la fórmula de cálculo del indicador y la relevancia de las evidencias solicitadas para evaluar el grado de cumplimiento con el indicador.

A partir de los resultados de validación realizada por los expertos realizamos la validez cuantitativa y cualitativa del modelo. La validez cuantitativa se realizó, calculando el índice de la validez facial, el índice de la validez de contenido y el índice de la fiabilidad interjueces para todas las dimensiones e indicadores del modelo. La validación cualitativa del modelo se llevó a cabo a partir de la recopilación de todos los comentarios realizados por los expertos que justificaban la razón de su validación y planteaban sugerencias para mejorar el modelo.

Como complemento a la validación cualitativa por expertos, se procedió al pilotaje del modelo. El objetivo era delimitar su fiabilidad, determinando el grado de consistencia interna del modelo, tanto para cada uno de los indicadores y de las dimensiones, así como para el conjunto total del modelo. Este pilotaje consistió en la autoevaluación de los cuatro programas de asignaturas ofrecidas por la Universidad Abierta y a Distancia de México (UnADM), reconocida internacionalmente por su trayectoria en la enseñanza virtual. La validación se efectuó a finales del 2015 durante una estancia de investigación y se ejecutó a través de los programas: Fundamentos de Administración, Comunicación Efectiva en Salud, Sistemas de Calidad, Comunicación y Gestión de Financiamiento. Los criterios de selección de los programas fueron:

Un modelo para la autoevaluación de la calidad de programas de educación universitaria virtual. Renata Marciniak y Joaquín Gairín Sallán. 
1. Modalidad virtual. Los programas elegidos se realizan completamente on-line.

2. Vigencia. Los programas deben haber sido aprobados en distintos periodos históricos y, además, estar vigentes hasta el 2015.

3. Pertenencia. Los programas escogidos deben pertenecer a distintas carreras; aumentando así la utilidad de los resultados de la autoevaluación.

4. Posibilidad de innovación. Los programas elegidos deben pertenecer a carreras que se encuentran en el proceso de innovación (cambio o modificación). Se busca así la utilidad de los resultados de la autoevaluación, ya que sus resultados pueden servir como insumo para enriquecer dicha innovación.

Los programas elegidos fueron los que mejor cumplieron todos los criterios de selección mencionados, ya que se desarrollan completamente en la modalidad virtual; son actualmente ofrecidos por la universidad y constituyen programas obligatorios para los estudiantes que cursan las carreras a las que pertenecen dichos programas; tienen el carácter troncal y, finalmente, todos los programas están el proceso de modificación.

Para evaluar los programas elegidos se obtuvo el acceso a la plataforma virtual de la UnADM, comprobando la idoneidad de la mayoría de los indicadores del modelo, que se completó con entrevistas en profundidad con todos los responsables de los programas evaluados e involucrados en su diseño y desarrollo. En total, se entrevistaron quince personas: el Responsable de la Carrera de Ingeniería y Transporte, el Responsable de Gestión y Administración de Pequeñas y Medianas Empresas, la Responsable de la Carrera de Educación para la Salud, la Responsable de la Carrera de Administración de Empresas Turísticas, la Administradora de la Plataforma Virtual de la División de Ciencias Sociales y Administrativas, la Asesora Académica de la Carrera de Ingeniería y Transporte, la Asesora Metodológica de la Carrera de Gestión y Administración de PYMES, la Responsable de Diseño y Desarrollo Curricular de la Universidad, y tres docentes en línea. Limitaciones temporales y del momento del estudio impidieron entrevistar a los estudiantes y conocer su nivel de satisfacción en relación con el proceso de enseñanza- aprendizaje, tal como se había planeado al principio de la investigación.

Como instrumentos de recogida de información, se construyeron cuatro protocolos de autoevaluación que sirvieron como pauta de observación y evaluación a rellenar por los investigadores.

Una vez terminada la aplicación piloto del modelo provisional I, se contrastaron los resultados de validación cuantitativa y cualitativa, así como los resultados de la aplicación piloto del modelo con la literatura especializada para cada dimensión e indicador del modelo (ver, como ejemplo, la Tabla 3), lo que permitió tomar decisiones sobre el mantenimiento, cambio o eliminación de una dimensión o indicador y, en consecuencia, elaborar el modelo provisional II (segundo borrador) para llevar a cabo la autoevaluación del programa formativo virtual.

Un modelo para la autoevaluación de la calidad de programas de educación universitaria virtual. Renata Marciniak y Joaquín Gairín Sallán. 
Indicador APEV-7: Accesibilidad y publicidad de los objetivos formativos

\begin{tabular}{|c|c|c|}
\hline \multicolumn{3}{|c|}{ Hallazgos encontrados por fuentes } \\
\hline Validación cuantitativa & Validación cualitativa & Teoría \\
\hline $\begin{array}{l}\text { Indicador con validación } \\
\text { muy alta en todos los } \\
\text { índices cuantitativos: } \\
\text { El índice Ía = } 1 \text { (la nota } \\
\text { máxima). El índice CVR' } \\
\text { promedio = } 1 \text { (la nota } \\
\text { máxima). El índice Kappa } \\
\text { de Fleiss promedio=0,78. }\end{array}$ & $\begin{array}{l}\text { UnADM: } \\
\text { La Universidad no utiliza el } \\
\text { indicador. } \\
\text { Expertos: } \\
\text { Indicador altamente valorado, sin } \\
\text { embargo no todos los expertos } \\
\text { entienden bien que significa } \\
\text { accesibilidad de objetivos } \\
\text { formativos. Además, recomiendan } \\
\text { añadir a las evidencias la página } \\
\text { web del programa como medio de } \\
\text { su difusión. }\end{array}$ & $\begin{array}{l}\text { Para Ardila (2011) los objetivos } \\
\text { del curso se deben dar a conocer } \\
\text { oportunamente. } \\
\text { Según la ANECA (2015) los } \\
\text { estudiantes matriculados en el } \\
\text { título, deben disponer de acceso } \\
\text { en el momento oportuno a la } \\
\text { información relevante del plan de } \\
\text { estudios y de los objetivos de } \\
\text { aprendizaje previstos. }\end{array}$ \\
\hline \multicolumn{3}{|c|}{ Decisiones de la investigadora: } \\
\hline $\begin{array}{l}\text { 1. Se ajusta la denominacic } \\
\text { Denominación: Los ob } \\
\text { 2. En los objetivos del indi } \\
\text { "disponibles". }\end{array}$ & $\begin{array}{l}\text { del indicador para favorecer la unive } \\
\text { ivos formativos están disponibles pa } \\
\text { dor y su descripción se reemplaza la }\end{array}$ & $\begin{array}{l}\text { cidad de la siguiente manera: } \\
\text { a todas las personas interesadas. } \\
\text { alabra "accesibles" por }\end{array}$ \\
\hline \multicolumn{3}{|c|}{$\begin{array}{l}\text { Evidencias: Página web de la universidad. Página web del programa virtual. Otras herramientas } \\
\text { de difusión del programa. }\end{array}$} \\
\hline
\end{tabular}

Tabla 3. Ejemplo de contrastación de los datos obtenidos para un indicador del modelo (elaboración propia)

Este modelo provisional II se validó a través de dos grupos de discusión: uno configurado con once expertos en la educación virtual y otro con trece usuarios (estudiantes) de la educación universitaria virtual. Ambos grupos se se desarrollaron en México, continuando el estudio de validación realizado en 2015, aunque una parte importante de los participantes del segundo grupo están cursando alguna carrera en línea en las universidades españolas. La validación se llevó a cabo en 2016.

El primer grupo de discusión estaba conformado por los expertos de la educación virtual ofrecida por el Sistema de Universidad Virtual de la Universidad de Guadalajara (UDGVirtual). Se eligió esta universidad, por ser la única que respondió a nuestra solicitud de llevar a cabo dicho grupo de las cinco invitadas. La sesión reunió a expertos elegidos por la Rectora de la UDGV, tratándose de las personas con más conocimiento y experiencia en la autoevaluación de la calidad de programas de educación universitaria virtual.

El segundo grupo de discusión se llevó a cabo entre usuarios de la educación virtual, concretamente entre los estudiantes que cursan alguna carrera en línea. El muestreo se realizó mediante la técnica de bola de nieve en la Universidad Panamericana Campus de Guadalajara (México), que dio permiso para realizar el grupo de discusión con los estudiantes que estudian en línea. Dicha técnica es una de las técnicas estadísticas de muestreo no aleatorios que se recomienda utilizar cuando surgen dificultades para identificar o tener acceso a la población objeto de estudio. El muestreo a través de dicha técnica se desarrolla de la siguiente manera:

Un modelo para la autoevaluación de la calidad de programas de educación universitaria virtual. Renata Marciniak y Joaquín Gairín Sallán.

Página 11 de 30 
"Partimos de individuos o grupos clave ya localizados y éstos, a su vez, nos conducen a otros con iguales características. Poco a poco, se aumenta el número de sujetos de la muestra (a través de las relaciones existentes entre los propios individuos) hasta conseguir una muestra suficiente" (Icart y Pulpón, 2012, p. 117).

Las características que debían cumplir los estudiantes/participantes en el grupo de discusión, fueron las siguientes:

- el estudiante debe estar realizando sus estudios (estudiante actual),

- el estudiante debe cursar estudios superiores,

- el estudiante debe cursar sus estudios totalmente en la modalidad virtual (e-learning puro),

- algunos de los estudiantes deben realizar sus estudios fuera de México.

La validación realizada por los participantes de los dos grupos nos permitió ajustar y mejorar el modelo a un conjunto de observaciones realizadas por ellos. Estas observaciones, que fueron incorporadas, nos sirvieron para elaborar el modelo definitivo que se presenta a continuación.

\section{Resultados: Propuesta del nuevo modelo}

El Modelo de Autoevaluación de Programas de Educación Universitaria Virtual definitivo está integrado por un total de 2 variables, 14 dimensiones, 3 subdimensiones y 111 indicadores de evaluación. En la Figura 1 se presenta la estructura del modelo.

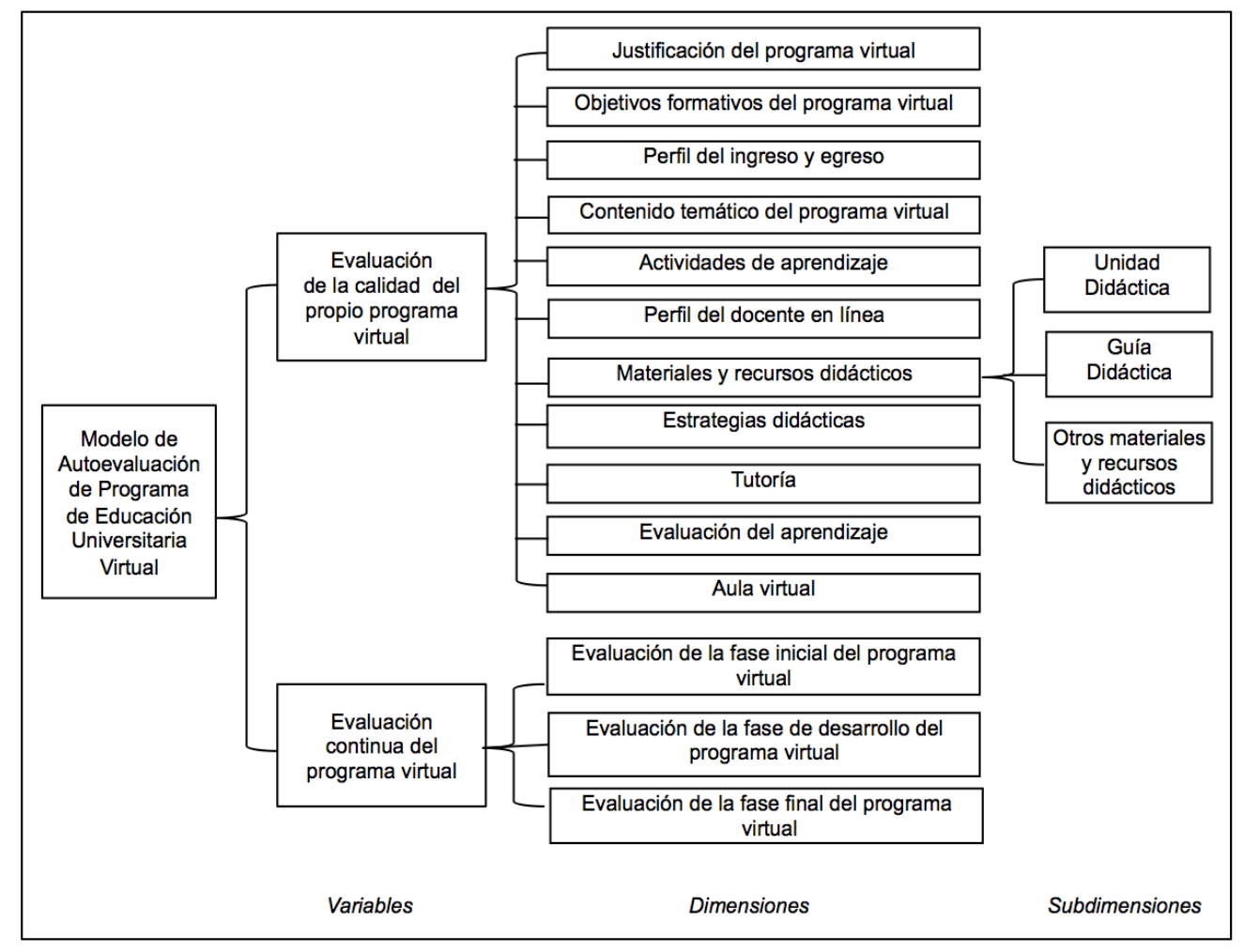

Figura 1. Estructura del Modelo de Autoevaluación de Programas de Educación Universitaria Virtual (elaboración propia)

Un modelo para la autoevaluación de la calidad de programas de educación universitaria virtual. Renata Marciniak y Joaquín Gairín Sallán. 
La primera variable a evaluar es la evaluación de la calidad del propio programa virtual. Consideramos que esta variable es fundamental, al permitir evaluar la calidad de todos los elementos que constituyen un programa formativo de una asignatura impartida bajo la modalidad virtual. Permite conocer el nivel de calidad tanto de los elementos pedagógicos como tecnológicos: justificación del programa, objetivos del programa, perfil de ingreso y egreso, contenido temático/temario del programa, actividades de aprendizaje, materiales y recursos didácticos, estrategias didácticas, estrategias de evaluación de aprendizaje, tutoría, aula virtual.

La segunda variable se refiere a la evaluación continua del programa virtual, que proporciona la información continua acerca del avance del mismo. Esta información permite mejorar el programa a través de la retroalimentación y autorregulación del mismo. La evaluación continua se debe realizar en tres momentos (fases) de realización del programa: la fase inicial, la fase de desarrollo y la fase final, con el objetivo de revisar, en un principio, lo que se había planificado, organizado, preparado para saber si se puede empezar a realizar el programa, cómo se ha ido desarrollando el programa y, finalmente, comprobar si los objetivos propuestos del programa han sido alcanzados (medición de los efectos).

La Tabla 4 presenta de una manera operativa todas las dimensiones del modelo.

\begin{tabular}{|c|c|}
\hline Dimensión & Definición operativa \\
\hline \multicolumn{2}{|c|}{ Variable 1: Evaluación de la calidad del propio programa virtual } \\
\hline $\begin{array}{l}\text { Justificación del } \\
\text { programa virtual }\end{array}$ & $\begin{array}{l}\text { Determinación de las razones de ser del programa virtual, haciendo referencia a } \\
\text { para qué el estudiante debe hacer el esfuerzo de realizar el programa. } \\
\text { Permite evaluar si se justifica: ¿por qué el programa virtual? }\end{array}$ \\
\hline $\begin{array}{l}\text { Objetivos } \\
\text { formativos del } \\
\text { programa virtual }\end{array}$ & $\begin{array}{l}\text { Declaración de los propósitos de aprendizaje que se pretende lograr con el } \\
\text { programa virtual. } \\
\text { Permite evaluar si se explica: ¿para qué el programa virtual? }\end{array}$ \\
\hline $\begin{array}{c}\text { Perfil de ingreso } \\
\text { y egreso }\end{array}$ & $\begin{array}{l}\text { Perfil de ingreso se entiende como un conjunto de conocimientos, habilidades y } \\
\text { actitudes que debe poseer el aspirante al programa para que lo curse. Perfil de } \\
\text { egreso define competencias que el estudiante adquirirá al terminar el programa. } \\
\text { Permite evaluar si se describen los destinatarios del programa y qué } \\
\text { competencias van a desarrollar. }\end{array}$ \\
\hline $\begin{array}{l}\text { Contenido } \\
\text { temático/ } \\
\text { temario del } \\
\text { programa virtual }\end{array}$ & $\begin{array}{l}\text { Presentación de los temas y subtemas que constituyen un programa virtual para } \\
\text { que el estudiante aborde, de manera general, la problemática que se le presentará } \\
\text { a lo largo de la realización del programa. } \\
\text { Permite evaluar qué se enseña. }\end{array}$ \\
\hline $\begin{array}{l}\text { Actividades de } \\
\text { aprendizaje }\end{array}$ & $\begin{array}{l}\text { Las diversas tareas mediante las cuales el docente en línea utiliza diversos } \\
\text { métodos, estrategias y técnicas de enseñanza para facilitar el aprendizaje en los } \\
\text { estudiantes. } \\
\text { Permite evaluar cómo se alcanzan los objetivos formativos del programa virtual. }\end{array}$ \\
\hline $\begin{array}{l}\text { Perfil del docente } \\
\text { en línea }\end{array}$ & $\begin{array}{l}\text { Conjunto de rasgos peculiares que caracterizan a la persona que realiza el } \\
\text { programa virtual. } \\
\text { Permite evaluar las competencias de las personas quienes realizan el programa } \\
\text { virtual. }\end{array}$ \\
\hline $\begin{array}{c}\text { Materiales y } \\
\text { recursos didácticos }\end{array}$ & $\begin{array}{l}\text { Cualquier material utilizado por el docente en línea o por el estudiante como } \\
\text { facilitador en el proceso de enseñanza-aprendizaje. } \\
\text { Permite evaluar la calidad de materiales con qué se realiza el programa virtual. }\end{array}$ \\
\hline $\begin{array}{l}\text { Estrategias } \\
\text { didácticas }\end{array}$ & $\begin{array}{l}\text { Las estrategias utilizadas por el docente en línea para apoyar el proceso de } \\
\text { enseñanza-aprendizaje y lograr los objetivos formativos del programa. } \\
\text { Permite evaluar el proceso educativo del programa virtual. }\end{array}$ \\
\hline & Proceso de acompañamiento a los estudiantes que lleva a cabo el docente en \\
\hline
\end{tabular}

Un modelo para la autoevaluación de la calidad de programas de educación universitaria virtual. Renata Marciniak y Joaquín Gairín Sallán.

Página 13 de 30 


\begin{tabular}{|c|l|}
\hline Tutoría & $\begin{array}{l}\text { línea; abarca, también, los procedimientos para ponerse en contacto con él. } \\
\text { Permite evaluar el acompañamiento y apoyo al estudiante durante la realización } \\
\text { de programa virtual. }\end{array}$ \\
\hline $\begin{array}{c}\text { Evaluación de } \\
\text { aprendizaje de los } \\
\text { estudiantes }\end{array}$ & $\begin{array}{l}\text { Estrategias y criterios utilizados por el docente en línea para evaluar el } \\
\text { aprendizaje de los estudiantes. } \\
\text { Permite evaluar cómo se valora lo que el estudiante ha aprendido. }\end{array}$ \\
\hline $\begin{array}{c}\text { Aula virtual del } \\
\text { programa }\end{array}$ & $\begin{array}{l}\text { Un software que permite llevar a cabo programas educativos en línea. išrṕp. } \\
\text { Permite evaluar la calidad del entorno virtual a través del cual se realiza el } \\
\text { programa. }\end{array}$ \\
\hline $\begin{array}{c}\text { Variable 2: Evaluación continua del programa } \\
\text { Evaluación de la } \\
\text { fase inicial del } \\
\text { programa virtual }\end{array}$ & $\begin{array}{l}\text { La primera fase por la que pasa el programa. } \\
\text { Permite evaluar lo que se había planificado, organizado, preparado para saber si } \\
\text { se puede empezar a realizar el programa. }\end{array}$ \\
\hline $\begin{array}{c}\text { Evaluación de la } \\
\text { fase de desarrollo } \\
\text { del programa } \\
\text { virtual }\end{array}$ & $\begin{array}{l}\text { La segunda fase por la que pasa el programa. } \\
\text { Permite evaluar cómo se ha ido desarrollando el programa. }\end{array}$ \\
\hline $\begin{array}{c}\text { Evaluación de la } \\
\text { fase final del } \\
\text { programa virtual }\end{array}$ & $\begin{array}{l}\text { La última fase por la que pasa el programa. } \\
\text { Permite evaluar los resultados del programa. }\end{array}$ \\
\hline
\end{tabular}

Tabla 4. Operacionalización de las dimensiones del modelo (elaboración propia a partir de Marciniak, 2016)

Además de las definiciones operativas de las dimensiones, se establecieron los requisitos de calidad que debe cumplir el programa dentro de cada dimensión del modelo para que sea considerado de alta calidad. Dichos requisitos son los siguientes:

- Dimensión 1: Justificación del programa virtual

Requisitos: El programa virtual debe cubrir las necesidades formativas y de mercado laboral, así como ser importante para que el estudiante lo encuentre razonable y de sentido al aprendizaje y desarrollo profesional.

- Dimensión 2: Objetivos formativos del programa

Requisitos: El programa virtual debe tener definidos unos objetivos formativos claros, medibles, coherentes con el temario del programa y que respondan a la demanda del mercado laboral; dichos objetivos deben estar disponibles y ser públicos para todas las personas interesadas en el programa virtual.

- Dimensión 3: Perfil de ingreso y egreso

Requisitos: El programa virtual debe definir de forma clara tanto el perfil de ingreso como de egreso. El perfil de ingreso debe presentar un conjunto de condiciones que debe cumplir el estudiante para ser admitido en el programa virtual. El perfil de egreso debe estar definido en términos de competencias cognitivas, procedimentales y actitudinales. En su elaboración deben participar distintos actores internos y externos. Los dos perfiles deben ser pertinentes a las necesidades del mercado laboral y accesibles a los estudiantes.

- Dimensión 4: Contenido temático/temario del programa virtual

Requisitos: El contenido temático del programa virtual debe ser adecuado a la carga lectiva del programa, y coherente con los objetivos formativos y el perfil de egreso; los temas y subtemas del temario deben ser actuales, fáciles de entender y ordenados en forma lógica.

Un modelo para la autoevaluación de la calidad de programas de educación universitaria virtual. Renata Marciniak y Joaquín Gairín Sallán. 
- Dimensión 5: Actividades de aprendizaje

Requisitos: Las actividades de aprendizaje del programa deben ser de distintos tipos y favorecer el logro de los objetivos formativos del programa virtual, trabajo colaborativo y adquisición de las competencias establecidas en el perfil de egreso del mismo. Asimismo, el programa debe ofrecer al estudiante directrices, cronograma, fechas y modo de entrega de todas las actividades para que tenga claro cómo y cuándo realizar dichas actividades y cómo y cuándo entregarlas al docente en línea.

- Dimensión 6: Perfil del docente en línea

Requisitos: El programa virtual debe estar desarrollado por el docente en línea que posea un perfil profesional adecuado para impartir el programa y unas competencias pedagógicas y tecnológicas adecuadas para llevar a cabo el proceso de enseñanzaaprendizaje en la modalidad virtual.

- Dimensión 7: Materiales y recursos didácticos

Requisitos: El programa debe contar con materiales y recursos didácticos de distintos tipos, adecuados y suficientes en cantidad y calidad, actualizados, motivadores y accesibles a los estudiantes. El material didáctico básico lo constituye la Unidad Didáctica que debe contener todos los contenidos del programa, estructurados y organizados para el aprendizaje. Dicha Unidad debe cumplir con todas las normas, estándares, directrices desarrollados para elaborar un material didáctico para un Entorno Virtual de Aprendizaje. Además, los estudiantes deben tener también a su disposición la Guía Didáctica que les brinda la información sobre qué van a estudiar, por qué, para qué, cómo, cuándo, con qué, con quiénes y cómo se va a evaluar lo que han aprendido. La Guía debe incluir toda la información necesaria para familiarizar al estudiante con todos los elementos del programa y el manejo provechoso de estos elementos para la construcción del aprendizaje significativo.

- Dimensión 8: Estrategias didácticas

Requisitos: Las estrategias de enseñanza empleadas por el docente en línea deben ser de distintos tipos y adecuadas al contenido temático del programa y modalidad virtual y ser coherentes con los objetivos formativos del programa. Además, el programa debe proponer un conjunto de estrategias de aprendizaje que el docente en línea promueve entre los estudiantes.

- Dimensión 9: Tutoría

Requisitos: El programa virtual debe contar con un sistema de tutoría eficaz y vías de comunicación claramente establecidas y accesibles a todos los estudiantes. Además, los estudiantes deben conocer las funciones del docente en línea y cómo y cuándo se pueden poner en contacto con él. Asimismo, el docente en línea debe realizar tutorías tanto grupales como individuales y proporcionar retroalimentación a los estudiantes tras cada actividad realizada por los mismos. También se debe llevar a cabo un seguimiento de las tutorías realizadas por el docente en línea y de manera permanente.

- Dimensión 10: Evaluación de aprendizaje de los estudiantes

Requisitos: El programa debe seguir normas y criterios claros y equitativas de evaluación continua de aprendizaje de los estudiantes y aplicar los mismos teniendo en cuenta la naturaleza de las distintas actividades de aprendizaje. Además, los estudiantes deben estar involucrados en el proceso de evaluación continua tanto de su propio aprendizaje como del de los otros estudiantes.

Un modelo para la autoevaluación de la calidad de programas de educación universitaria virtual. Renata Marciniak y Joaquín Gairín Sallán.

Página 15 de 30 
- Dimensión 11: Aula virtual

Requisitos: El programa debe contar con un aula virtual que posibilite gestionar todas sus fases: desde la elaboración de los contenidos, su distribución o puesta en línea y uso, hasta la evaluación de las competencias adquiridas por el estudiante o la evaluación de su proceso formativo.

- Dimensión 12: Evaluación de la fase inicial del programa virtual

Requisitos: El programa virtual debe ser evaluado una semana (como mínimo) antes de su comienzo para establecer el grado de su preparación para su inicio.

- Dimensión 13: Evaluación de la fase de desarrollo del programa virtual

Requisitos: El programa virtual debe ser evaluado a la mitad de su realización para identificar los puntos débiles del mismo en su desarrollo y tomar decisiones para eliminarlos.

- Dimensión 14: Evaluación de la fase final del programa virtual

Requisitos: El programa virtual debe ser evaluado inmediatamente después de su finalización para medir el grado de consecución de los objetivos formativos, los resultados del programa y la satisfacción de los estudiantes con el programa terminado.

\section{Indicadores del modelo}

Tras definir la estructura del modelo para llevar a cabo la autoevaluación de programas de educación universitaria virtual, delimitar sus principales dimensiones y requisitos para cada una de ellas, nos vimos obligados a afrontar el gran reto de diseñar un sistema de indicadores de calidad para medir cada una de las catorce dimensiones propuestas en el modelo. Con este fin, nuevamente, se analizaron los estándares, normas, directrices, modelos, guías y otras herramientas existentes (ISO/IEC 19796-1:2005; CWA 15660:2007; UNIQUe, 2014; UNE 66181:2012; ADL, 2014) el modelo de los cuatro niveles de Kirkpatrick, el modelo sistémico de Vann Slyke, el modelo de los cinco niveles de evaluación de Marshall, modelo LORI, E-learning Maturity Model, SEVAQ+ Self-Evaluation Tool for eLearning, EFMD CEL Guide to Self Assessment, Guide to Evaluating Distance Education and Correspondence Education, GUÍA @FORTIC, entre otros), constatando en ellos la presencia de los indicadores que nos permitieran evaluar las dimensiones de nuestro modelo.

Como resultado de la antemencionada investigación bibliográfica, se elaboró un total de 111 indicadores que facilitan la identificación de la calidad del programa virtual de cada una de las dimensiones propuestas para el modelo. La Tabla 5 presenta la distribución de los indicadores según las dimensiones y en el Anexo 1 se presentan los indicadores elegidos para evaluar cada una de estas dimensiones.

Un modelo para la autoevaluación de la calidad de programas de educación universitaria virtual. Renata Marciniak y Joaquín Gairín Sallán. 


\begin{tabular}{|c|c|}
\hline Dimensiones & $\begin{array}{l}\text { Número de } \\
\text { indicadores }\end{array}$ \\
\hline \multicolumn{2}{|l|}{$\begin{array}{l}\text { VARIABLE 1: EVALUACIÓN DE LA CALIDAD DEL PROPIO PROGRAMA } \\
\text { VIRTUAL }\end{array}$} \\
\hline Justificación del programa virtual & 3 \\
\hline Objetivos formativos del programa virtual & 5 \\
\hline Perfil del estudiante & 7 \\
\hline Contenido temático/ temario del programa virtual & 5 \\
\hline Actividades de aprendizaje & 8 \\
\hline Perfil del docente en línea & 3 \\
\hline Materiales y recursos didácticos & 38 \\
\hline Estrategias didácticas & 3 \\
\hline Tutoría & 7 \\
\hline Evaluación del aprendizaje de los estudiantes & 4 \\
\hline Calidad del aula virtual del programa & 9 \\
\hline \multicolumn{2}{|l|}{ VARIABLE 2: EVALUACIÓN CONTINUA DEL PROGRAMA VIRTUAL } \\
\hline Evaluación de la fase inicial del programa & 4 \\
\hline Evaluación de la fase de desarrollo del programa & 7 \\
\hline Evaluación de la fase final del programa & 8 \\
\hline TOTAL & 111 \\
\hline
\end{tabular}

Tabla 5. Distribución de los indicadores del modelo según las dimensiones

Para el diseño del sistema de indicadores se tuvieron en cuenta las directrices para elaborar un sistema de indicadores establecidas por la Asociación Española de Normalización y Certificación (AENOR, 2012) en la norma UNE 66175:2012. En dicha norma podemos leer, entre otros:

"Un indicador no debe dar lugar a interpretaciones diferentes, por ello para conseguir este objetivo, cada usuario deberá aplicar los criterios aquí indicados en función de sus necesidades. Los conceptos que pueden considerarse a la hora de definir los indicadores son los siguientes:

- selección del indicador,

- denominación del indicador,

- forma de cálculo: su especificación y fuentes de información,

- forma de representación,

- definición de responsabilidades,

- definición de umbrales y objetivos” (p. 9).

Para facilitar la aplicación y precisar la utilidad de los indicadores propuestos, se elaboró una ficha descriptiva para cada uno de ellos que contiene los elementos indicados por la norma UNE 66175 y dos elementos adicionales: código y descripción del indicador. La tabla 6 presenta un ejemplo de ficha descriptiva de indicador.

Un modelo para la autoevaluación de la calidad de programas de educación universitaria virtual. Renata Marciniak y Joaquín Gairín Sallán. 


\begin{tabular}{|l|l|}
\hline Código & APEV-1 \\
\hline Denominación & Respuesta a las necesidades formativas. \\
\hline Objetivo & $\begin{array}{l}\text { Constatar que el programa virtual ha sido creado en relación con las necesidades de } \\
\text { formación de la región de influencia de la universidad. }\end{array}$ \\
\hline Descripción & $\begin{array}{l}\text { El programa formativo virtual se justifica si da respuesta a una necesidad formativa } \\
\text { en la modalidad virtual. Para el Comité de diseño del programa, las necesidades } \\
\text { formativas deben constituir la base de elección de una asignatura y dar respuesta a la } \\
\text { pregunta ipor qué esa y no otra asignatura? }\end{array}$ \\
\hline $\begin{array}{l}\text { Fórmula de } \\
\text { cálculo }\end{array}$ & $\begin{array}{l}\text { Opción: SÍ/NO } \\
\text { Niveles de medición: la respuesta SÍ equivale a 4 puntos, la respuesta NO equivale a } \\
1 \text { punto. }\end{array}$ \\
\hline Evidencias & $\begin{array}{l}\text { Un documento que justifique el programa. Entrevista con el Responsable del } \\
\text { Programa. Estudios actualizados sobre las necesidades formativas en la región de } \\
\text { influencia de la universidad. }\end{array}$ \\
\hline $\begin{array}{l}\text { Forma de } \\
\text { presentación }\end{array}$ & Protocolo de autoevaluación del programa virtual. \\
\hline Responsables & Comité de Autoevaluación de Programa Virtual. \\
\hline
\end{tabular}

Tabla 6. Ficha descriptiva del indicador APEV-1 (elaboración propia)

\section{Validación del modelo}

Más allá del proceso amplio desarrollado en la construcción del modelo, se presenta a continuación un resumen de los resultados de la validación cualitativa de los indicadores realizada por expertos y de acuerdo con los criterios de univocidad, pertinencia, importancia, apropiación de fórmula de cálculo y relevancia de evidencia, así como los comentarios generales recogidos para la definición de los propios indicadores.

Los resultados de la validación indican que todos los indicadores fueron valorados por los expertos como unívocos linguísticamente y pertinentes en el contenido para las dimensiones en las que fueron incluidos. Respecto del criterio "importancia", nueve indicadores de los 120 fueron eliminados porque, a juicio de los expertos, no aportaban información para evaluar la calidad del programa. Estos indicadores fueron: «Adecuación de las actividades de aprendizaje a la modalidad virtual», «Requisitos de conocimiento y uso de las TICs del aspirante al programa virtual», «Criterios de selección de contenidos temáticos del programa virtual, «Capacitación del docente en línea en cuanto a la educación virtual», «Capacitación de las personas responsables de desarrollo del programa en cuanto a la educación virtual», «Adecuación de materiales y recursos didácticos para las personas con necesidades especiales», «Variedad de materiales didácticos», «Variedad de recursos didácticos», «Elementos interactivos de contenidos de la Unidad Didáctica».

Todas las fórmulas fueron valoradas como apropiadas en relación al criterio de valoración "apropiación de fórmula de cálculo". No obstante, los comentarios efectuados a los indicadores sugirieron, en términos generales, mejorar algunos de ellos y reformular las evidencias solicitadas, a pesar de que todas ellas fueron valoradas como relevantes o altamente relevantes.

Todos los comentarios realizados por los expertos relacionados con los indicadores del modelo se pueden, desde la generalidad, resumir en las siguientes sugerencias:

Un modelo para la autoevaluación de la calidad de programas de educación universitaria virtual. Renata Marciniak y Joaquín Gairín Sallán. 
- Modificar la denominación de determinados indicadores por conceptos más comprensibles para los destinatarios finales del modelo.

- Mejorar las definiciones operativas de algunos indicadores.

- Suprimir del modelo algunos indicadores que no son signos de calidad ni de éxito de un programa virtual.

- Añadir, explicar mejor o cambiar algunas evidencias solicitadas para determinados indicadores.

- Precisar más o cambiar las fórmulas de cálculo para determinados indicadores; especificar cuantitativamente los valores de la escala de cálculo.

La validación realizada por los expertos nos permitió ajustar y mejorar el modelo conforme con los comentarios realizados por los mismos.

\title{
Discusión y conclusiones
}

La autoevaluación es una forma de identificar las fortalezas y debilidades de un programa formativo impartido bajo la modalidad virtual y mejorar su calidad.

\begin{abstract}
"Es un proceso a través del cual la comunidad universitaria directamente implicada en una enseñanza ha de reflexionar, describir, analizar, valorar la realidad de la misma, basando sus afirmaciones en datos objetivos, y determinar las propuestas de mejora que ha de implementar para superar las debilidades" (ANECA, s.f., p. 10).
\end{abstract}

En opinión de Gairín et al. (2014), la realización de la autoevaluación de programas formativos viene justificada por la responsabilidad que tienen los profesionales sobre la mejora de la universidad y de los programas que en ellos se desarrollan. Sin embargo, la educación virtual es tan diferente en su organización, inscripción y operación de la educación presencial que no se puede llevar a cabo la autoevaluación de programas formativos impartidos en estas dos modalidades (presencial y virtual) de la misma manera y utilizando modelos iguales. Según muchos autores (Jung y Latchem, 2012; Marciniak, 2015; Martínez, 2013; Silvio, 2006; Veytia y Chao, 2013), la calidad de la educación virtual no es comparable con - ni puede reducirse a - los criterios otorgados para la educación presencial. Requiere criterios, metodologías particulares y modelos de evaluación adecuados al objeto y a los distintos contextos en los que se produce y desarrolla este tipo de educación.

Diseñar un modelo que sea adecuado para evaluar la calidad de un programa formativo impartido bajo la modalidad virtual es una tarea compleja, que debe tener en cuenta la posibilidad de evaluar la calidad del propio programa (Martínez, 2013), así como la evaluación continua del mismo, tal y como postula Sarramona (2001):

"La evaluación de un programa de educación a distancia ha de abarcar evaluación desde el diagnóstico previo (el diagnóstico de necesidades y contexto) hasta la planificación (la evaluación del «input», es decir, de los elementos que constituyen el programa del curso: contenidos, recursos didácticos, estrategias de evaluación, etc.), el proceso aplicativo (respecto a los posibles ajustes a realizar para que el curso cumpla sus propósitos), los resultados (previstos, indirectos y no previstos); la evaluación a corto y largo plazo (todos los resultados deben ser evaluados en perspectiva temporal), los formadores (p. 6).

Un modelo para la autoevaluación de la calidad de programas de educación universitaria virtual. Renata Marciniak y Joaquín Gairín Sallán.

Página 19 de 30 
Teniendo en cuenta estas indicaciones, el modelo de autoevaluación que se propone en este trabajo integra la evaluación de todos los elementos que constituyen un programa formativo virtual (evaluación de la calidad del propio programa) con la evaluación continua del mismo, es decir, las tres fases por las que el programa pasa durante su existencia.

El modelo abarca catorce dimensiones, de las cuales once sirven para evaluar el nivel de calidad de los elementos pedagógicos y tecnológicos que configuran el programa y tres dimensiones sobre el proceso, tal y como se puede ver en la tabla 3.

Comparando nuestro modelo con otros propuestos podemos deducir que tiene algunos aspectos comunes y a la vez integra dimensiones divergentes. Entre dimensiones iguales, aunque algunas con diferente denominación, se encuentran: perfil de estudiantes, perfil de docentes, evaluación de la fase de desarrollo del programa y su fase final (resultados). Sin embargo, al estudiar exhaustivamente las dimensiones de nuestro modelo y de las propuestas por diferentes modelos se notan los diferentes significados asignados a cada una por sus autores. Por ejemplo, en la dimensión referida a los estudiantes encontramos dos grupos de enfoques. El primer grupo lo constituyen los modelos que proponen evaluar las características de los destinatarios (Oliver, 2001; EFMD, 2006) como sus habilidades tecnológicas, acceso a la tecnología, autoregulación de aprendizaje, etc. El segundo grupo lo conforman los modelos que proyectan evaluar la satisfacción de los estudiantes (Kirkpatrick, 2007; CALED, 2010; EFQUEL, 2010). Nosotros coincidimos con el primer grupo de los autores y proponemos la dimensión "perfil del estudiante" que abarca dos sub-dimensiones: "Perfil de ingreso" y "Perfil de egreso" y ofrecemos indicadores que permiten, entre otros, evaluar si los dos perfiles están bien definidos y disponibles para las personas interesadas.

En cuanto a la dimensión "Perfil de los docentes", nuestro modelo no se diferencia de las propuestas por otros autores (Marshall, 2007), ya que también propone evaluar si el programa virtual está desarrollado por el docente en línea que posee el perfil profesional adecuado para impartir el programa y las competencias pedagógicas y tecnológicas adecuadas para llevar a cabo el proceso de enseñanza-aprendizaje en la modalidad virtual.

Con referencia a la dimensión "Evaluación de la fase de desarrollo del programa" se nota un desacuerdo entre los autores en cuanto a los aspectos que se deben evaluar dentro de esta dimensión. Algunos autores, cuando hablan de desarrollo, se refieren a la autorización y producción de los materiales y recursos didácticos (Marshall, 2007). Para Marcelo y Zapata (2008), la dimensión desarrollo del programa de formación (denominada también como implementación) "tiene que ver con la fase y los procedimientos en los que se utilizan los recursos técnicos y humanos necesarios, mediante estrategias formativas variadas que favorecen la consecución de las metas y objetivos del programa" (p. 17). Para otros, la evaluación del desarrollo de programa se refiere a la evaluación a la mitad de su realización (SEA, 2010). Nosotros coincidimos con este último grupo de autores. No obstante, nuestro modelo se difiere de las propuestas de dichos autores, ya que hace énfasis en la identificación de los puntos débiles de la etapa de procesual del programa y tomar decisiones para eliminarlos.

En la esfera de la evaluación de "resultados" también encontramos una diversidad de enfoques en los modelos analizados que responden a paradigmas, algunas veces, contradictorios y opuestos. Por ejemplo, García Aretio (1998), para la evaluación de los Un modelo para la autoevaluación de la calidad de programas de educación universitaria virtual. Renata Marciniak y Joaquín Gairín Sallán.

Página 20 de 30 
resultados propone indicadores referidos al redimiendo académico, mientras que Marshall (2007) habla del grado en el que el curso on-line permite a los participantes transferir los conocimientos adquiridos al puesto de trabajo. Según la University of Wisconsin (UW, 2002), la evaluación de los resultados se refiere a la evaluación de los cambios de los individuos, grupos, comunidades, organizaciones, comunidades o sistemas, mientras que para la African Virtual University (AVU, 2014) es la evaluación de la retención de los estudiantes, tasa de rendimiento, e impacto de programa. Nuestro modelo propone la dimensión "Evaluación de la fase final del programa" que abarca indicadores que permiten medir el grado de consecución de los objetivos formativos, los resultados del programa y la satisfacción de los estudiantes con el programa terminado.

Los modelos desarrollados para evaluar la calidad de educación virtual (AVU, 2014; García Aretio, 1998; Sarramona, 2001; Shelton y Moore, 2011; Zhang y Jiang, 2012), proponen también evaluar algunos aspectos relacionados con la "pedagogía" tales como: objetivos formativos, materiales y recursos didácticos, actividades, estrategias de evaluación. Nuestro modelo igualmente propone evaluar dichos aspectos pero la diferencia radica en que nuestro modelo abarca todos estos aspectos, y los otros, sólo algunos de ellos. Estos aspectos y otros que raramente se propone evaluar como: justificación del programa, contenidos temáticos, tutoría, estrategias didácticas, y aula virtual, definen la calidad del propio programa virtual y por eso deberían ser incluidos en un modelo que pretende evaluar dicha calidad. Si un modelo propone sólo algunas de estas dimensiones, la evaluación es incompleta y no permite una diagnosis profunda de la calidad de programa actual y realizar una reflexión sobre qué es necesario cambiar y qué no, para asegurar dicha calidad de alto nivel.

Otra cosa que diferencia nuestro modelo de otras propuestas es el sistema de indicadores que lo compone y las fichas descriptivas elaboradas para cada indicador que permiten comprobar si el programa cumple con el indicador. Así, se ayuda a los responsables de la realización de la autoevaluación de los mencionados programas a la hora de llevar a cabo dicho proceso, ya que dichas fichas constituyen una guía de autoevaluación indicando qué, por qué y cómo evaluar el cumplimiento del indicador.

El modelo está dirigido a los responsables de la ejecución de programas formativos impartidos bajo la modalidad virtual, directores/coordinadores de las carreras a las que pertenece el programa. Asimismo, puede servir a los diseñadores de programas virtuales como pauta para diseñar un programa virtual de buena calidad.

\section{Limitaciones del estudio y líneas de investigación futuras}

"En todos los procesos de investigación es habitual encontrar limitaciones que requerirán del investigador o de la investigadora un espíritu de superación para resolver los objetivos del trabajo iniciado" (Khalil, 2013, p. 413). Las principales limitaciones que hemos encontrado en el proceso de diseño del modelo son las siguientes:

- Escasez bibliográfica de estudios sobre autoevaluación de programas de educación universitaria virtual, que conllevó la necesidad de extrapolar la información existente sobre el tema del campo de la evaluación de educación tradicional.

Un modelo para la autoevaluación de la calidad de programas de educación universitaria virtual. Renata Marciniak y Joaquín Gairín Sallán. 
- Los indicadores elegidos para el modelo son una selección dentro de los múltiples indicadores diseñados para evaluar la calidad de educación virtual en general, elegidos en nuestro caso a partir de su relevancia e importancia desde el punto de vista de los investigadores que han realizado el estudio y los que han participado en su validación.

- Otra limitación de la investigación está relacionada con el alcance de la posibilidad de la aplicación del modelo propuesto. Aunque nuestra intención fue diseñar un modelo flexible, que pueda ser utilizado por los responsables de la ejecución de programas formativos en diferentes países, la ley vigente en algunos países en materia de la educación virtual o los reglamentos internos de las universidades impiden el uso de ciertos indicadores o indican otros que no están en el modelo. Por lo tanto, se aconseja que las personas interesadas en el uso del modelo lo apliquen después de ajustarlo a los propios contextos de funcionamiento de la universidad y las propias necesidades de mejora de programas virtuales.

De todas formas, las limitaciones señaladas no han impedido el desarrollo del modelo ni limitado su rigurosidad. Estas limitaciones indican el marco para las futuras investigaciones en cuanto a la identificación de los elementos estables del modelo y aquéllos que deben ser adaptados al contexto específico de cada universidad. Además, dada la variabilidad de las condiciones de la calidad de la educación virtual, la necesidad de investigar el ámbito de las dimensiones y los indicadores, para ajustar el Modelo de Autoevaluación de Programas de Educación Universitaria Virtual a ellos, resulta siempre actual y justificada.

Presentación del artículo: 6 de abril de 2017

Fecha de aprobación: 30 de mayo de 2017

Fecha de publicación: 30 de junio de 2017

Marciniak, R., y Gairín, J. (2017). Un modelo para la autoevaluación de la calidad de programas de educación universitaria virtual. RED. Revista de Educación a Distancia, 54. Consultado el (dd/mm/aaaa) en http://www.um.es/ead/red/

\section{Financiación}

Esta investigación no ha recibido ninguna subvención específica de los organismos de financiación en los sectores públicos, comerciales o sin fines de lucro.

Un modelo para la autoevaluación de la calidad de programas de educación universitaria virtual. Renata Marciniak y Joaquín Gairín Sallán. 


\section{Referencias bibliográficas}

Abdulaziz, I. A., Almetair, N. A. y Nasser, M. A. (2014). Evaluation Models for ELearning Platform in Riyadh City Universities (RCU) with Applied of Geographical Information System (GIS). Asia Pacific Journal of Multidisciplinary Research, 2(6), 1-11.

ADL (Advanced Distributed Learning). (2014). SCORM. Shareable Content Object Reference Model. Recuperado de http://www.adlnet.gov/scorm/

AENOR (Asociación Española de Normalización y Certificación). (2012). Norma española UNE 66178: Sistema de gestión de la calidad. Guía para la implementación de sistemas de indicadores. Madrid: AENOR.

AICCJC (Accrediting Commission for Community and Junior Colleges). (2013). The Guide to Evaluating Distance Education and Correspondence Education. Recuperado de http://www.sdmiramar.edu/webfm_send/11848

ANECA (Agencia Nacional de Evaluación de la Calidad y Acreditación). (s.f.). Programa de evaluación institucional. Guía de autoevaluación (con adaptaciones específicas para la UNED). Recuperado de www.aneca.es

Attwell, G. (2006). Evaluating E-learning. A Guide to the Evaluation of E-learning. Bremen: Perspektiven-Offset-Druck. Recuperado de http://pontydysgu.org/wpcontent/uploads/2007/11/eva_europe_vol2_prefinal.pdf

AVU (African Virtual University). (2014). Design and Development of a Quality Assurance Framework. Recuperado de http://www.avu.org

Barnard, D. y Echols, J. (2015). The Anatomy of K-12 Online Programs: Practical Ideas and Guidelines. London: Rowman and Littlefield.

Bautista, J. R., Martínez, R. y Sainz, M. (2001). La evaluación de materiales didácticos para la educación a distancia. RIED Revista Iberoamericana de Educación a Distancia, 4(1), 73-95. Recuperado de http://revistas.uned.es/index.php/ried/article/viewFile/1192/1095

Boettcher, J. V. y Conrad, R. M. (2016). The Online Teaching Survival Guide: Simple and Practical Pedagogical Tips. London: John Wiley and Sons.

Butcher, N. y Wilson-Strydom, M. (2013). A guide to quality in online learning. Dallas TX: Academic Partnerships.

CALED (Instituto Latinoamericano y del Caribe de Calidad en Educación Superior a Distancia). (2010). Guía de autoevaluación para programas de pregrado a distancia. Loja: CALED.

CEN (Comité Europeo de Normalización). (2007). CWA 15660:2007, Providing good practice for E-Learning quality approaches. Brussels: CEN.

Chmielewski, K. (2013). Diagnoza stanu kształcenia na odległość w Polsce $i$ wybranych krajach UE [Diagnosis de la educación a distancia en Polonia y algunos países de la Unión Europea]. Warszawa: Demos Polska.

CIEES (Comités Interinstitucionales para la Evaluación de la Educación Superior). (2008). Metodología General para la Evaluación de Programas de Educación Superior a Distancia. México D.F.: CIEES.

Un modelo para la autoevaluación de la calidad de programas de educación universitaria virtual. Renata Marciniak y Joaquín Gairín Sallán.

Página 23 de 30 
Díaz-Maroto, I. T. (2009). Formación a través de internet: evaluación de la calidad. Barcelona: UOC.

Domínguez Castillo, J. G. y Morcillo Baquedano, J. S. (2016). Evaluación de un curso en línea para la formación de competencias en el uso de las TIC en profesores de ciencias de secundarias públicas del sureste de México. RED. Revista de Educación a Distancia, 51. Recuperado de DOI: http://dx.doi.org/10.6018/red/51/2

EADTU (European Association of Distance Teaching Universities). (2016). Quality Assessment for E-learning: a Benchmarking Approach. Third edition. Maastricht: EADTU.

EFMD (European Foundation for Management Development). (2006). EFMD CEL Criteria, Indicators and Standards. Recuperado de https://www.efmd.org/accreditation-main/equis/116-accreditation/cel

ENQA (European Association for Quality Assurance in Higher Education). (2015). Standards and Guidelines for Quality Assurance in the European Higher Education Area. 3rd edition. Brussles: EURASHE.

EFQUEL (European Foundation for Quality in eLearning). (2010). Handbook y Quality Criteria Toolkits: Open ECBCheck Initiative. Recuperado de https://quality4digitallearning.org/open-house/handbook-quality-criteria-toolkitsopen-ecbcheck-initiative/

Ehlers, U. (2013). Open Learning Cultures: A Guide to Quality, Evaluation, and Assessment for Future Learning. Berlin: Springer-Verlag.

Ejarque González, E., Buendía García, F. y Hervás Jorge, A. (2008). Aplicación de un modelo de calidad para evaluar experiencias e-learning en el Espacio Europeo Universitario. EDUCAR, 41, 11-28.

Fainholc, B. (2004). La calidad en la educación a distancia continúa siendo un tema muy complejo. RED. Revista de educación a distancia, 12. Recuperado de http://revistas.um.es/red/article/view/25311/24591

Fidalgo, A. (2007). Micro Guía de Indicadores para el elearning. Madrid: Universidad Politécnica de Madrid.

Gairín Sallán, J., Díaz-Vicario, A., Rosales Acín, M. y Sentinella Solé, X. (2014). La autoevaluación para la mejora de la seguridad integral en centros educativos. EDUCAR, 50(2), 363-381.

García Aretio, L. (2014). Bases, mediaciones y futuro de la educación a distancia en la sociedad digital. Madrid, Sintesis.

García Aretio, L. (1998). Indicadores para la evaluación de la enseñanza en una universidad a distancia. RIED - Revista Iberoamericana de Educación a Distancia. 1(1), 63-86. Recuperado de http://ried.utpl.edu.ec/sites/default/files/files/pdf/v\%201-1/volumen1-1.pdf

Giorgetti, C. G., Romero, L. y Vera, M. (2013). Diseño de un modelo de evaluación de la calidad específico para EaD. Revista de Universidad y Sociedad del Conocimiento (RUSC), 10(2), 54-68. Recuperado de https://link.springer.com/article/10.7238/rusc.v10i2.1742

Grifoll, J., Huertas, E., Prades, A., Rodríguez, S., Rubin, Y., Mulder, F. y Ossiannilsson, E. (2010). Quality Assurance of E-learning. Helsinki: ENQA.

Un modelo para la autoevaluación de la calidad de programas de educación universitaria virtual. Renata Marciniak y Joaquín Gairín Sallán. 
HECQ (Higher Education Compliance and Quality). (1997). Guidelines for Distance Education. London: HECQ.

Hilera González, J. R., y Hoya Marin, R. (2010). Estándares de e-learning: guía de consulta. Alcalá: Universidad de Alcalá.

Icart Isern M. T. y Pulpón Segura, A. M. (2012). Cómo elaborar y presentar un proyecto de investigación, una tesina y una tesis. Barcelona: Universidad de Barcelona.

ISO/IEC 19796-1:2005, Information technology -- Learning, education and training Quality management, assurance and metrics -- Part 1: General approach.

IEEE (Institute of Electrical and Electronics Engineers). (2014). Accredited Learning Technology Standards. Recuperado de https://ieee-sa.centraldesktop.com/ltsc/

Jung, I. y Latchem, C. (2012). Quality Assurance and Accreditation in Distance Education and e-Learning. New York/London: Routledge.

Khalil Hamdan, A. H. (2013). Construcción de un modelo de evaluación de la calidad de la enseñanza universitaria desde el punto de vista de los alumnos (Tesis doctoral). Universidad de Córdoba: Córdoba.

Kirkpatrick, D. L. (2007). Evaluación de acciones formativas: los cuatro niveles. $3^{\text {ra }}$ edición. Barcelona: Gestión 2000.

Latchem, C. (2014). Quality Assurance in Online Distance Education. En: O. ZawackiRichter y T. Anderson (Eds.). Online distance education. Towards a Research Agenda, pp. 132-156. Edmonton: AU Press.

Marcelo, C. y Zapata, M. (2008). Cuestionario para la evaluación: "Evaluación de la calidad para programas completos de formación docente a través de estrategias de aprendizaje abierto y a distancia". Metodología de uso y descripción de indicadores. RED, Revista de Educación a Distancia. Número monográfico VII, 131. Rcuperado de http://www.um.es/ead/red/M7/

Marciniak, R. (2016). Autoevaluación de programas de educación universitaria virtual (Tesis doctoral). Universitat Autònoma de Barcelona: Barcelona.

Marciniak, R. (2015). A methodological proposal for applying international benchmarking to evaluating the quality of higher virtual education. RUSC. Universities and Knowledge Society Journal, 12(2), 46-60. Recuperado de http://rusc.uoc.edu/rusc/ca/index.php/rusc/article/view/v12n3-marciniak/2650.html

Marciniak, R. y Gairín Sallán, J. (2017). Dimensiones de evaluación de calidad de educación virtual: Revisión de modelos referentes. RIED. Revista Iberoamericana de Educación a Distancia, 20(2), [en prensa].

Marshall, S. (2007). Development and Evolution of an E-Learning Maturity Model.

Recuperado de http://www.cad.vuw.ac.nz/wiki/images/a/a9/2006EducaMarshalleMMAbstract.pdf

Martínez Mediano, C. (2013). Evaluación de programas. Modelos y procedimientos. Madrid: UNED.

Masoumi, D. y Lindström, B. (2012). Quality in e- learning: a framework for promoting and assuring quality in virtual institutions. Journal of Computer Assisted Learning, 28(1), 27- 41.

Un modelo para la autoevaluación de la calidad de programas de educación universitaria virtual. Renata Marciniak y Joaquín Gairín Sallán. 
Mery, Y. y Newby, J. (2014). Online by Design: The Essentials of Creating Information Literacy Courses. Plymouth: Rowman y Littlefield.

Meza, J. (2012). Modelo pedagógico para proyectos de formación virtual. Bonn: GIZ.

Motz, R. (2013). Informe de análisis de estándares, normas y modelos de capacidad de madurez relacionados con la calidad y accesibilidad de la educación virtual. Recuperado de http://www.esvial.org/wp-content/files/E311_vf_v1.pdf

MSCHE (Middle States Commission on Higher Education). (2011). Distance education programmes: Guidelines for the Evaluation of Distance Education Programmes (Online Learning). Philadelphia: MSCHE.

Nesbit, J. C., Belfer, K. y Vargo, J. (2002). A convergent participation model for evaluation of learning objects. Canadian Journal of Learning and Technology. 28(3), 105-120.

OLC (Online Learning Consortium). (2011). The OLC Quality Scorecard for the Administration of Online Programs. Recuperado de https://onlinelearningconsortium.org/consult/olc-quality-scorecard-administrationonline-programs/

Oliver, R. G. (2001). Assuring the quality of online learning in Australian higher education. Proceedings of 2000 Moving Online Conference, 222-231.

Quesada Pallarès, C., Espona Barcons, B., Ciraso Calí, A. y Pineda Herrero, P. (2015). La eficacia de la formación de los trabajadores de la administración pública española: comparando la formación presencial con el eLearning. Revista del CLAD Reforma y Democracia, 6(1), 107-132.

Rausaria, R. R. y Lele, N. A. (2002). Self-Assessment of Distance Education Institutions: Identification of Parameters for Programme Assessment. Indian Journal of Open Learning, 11(1), 147-157.

Rodríguez-Santero, J. y Torres-Gordillo, J. J. (2016). La evaluación de cursos de formación online: el caso ISTAS. RED. Revista de Educación a Distancia, 49(9). Recuperado de en http://www.um.es/ead/red/49

Ruiz-Bolívar, C. y Dávila, A. A. (2016). Propuesta de buenas prácticas de educación virtual en el contexto universitario. RED. Revista de Educación a Distancia, 49(12). Recuperado de http://www.um.es/ead/red/49

Rushby, N. y Surry, D. 2016. Wiley Handbook of Learning Technology. New York, John Wiley and Sons.

Sarramona, J. (2001). Evaluación de programas de educación a distancia. RIED Revista Iberoamericana de Educación a Distancia, 1(1), 1-24. Recuperado de http://revistas.uned.es/index.php/ried/article/view/1189

SEA (Stowarzyszenie e-learningu Akademickiego) [Asociación de E-learning Académico de Polonia]. (2010). Kryteria oceny kursu/programu e-learningowego [Criterios de evaluación del curso/programa virtual]. Recuperado de http://www.sea.edu.pl/kryteria/?page=obszary

Shattuck, K. (2014). Assuring Quality in Online Education: Practices and Processes at the Teaching, Resource, and Program Levels. Sterling: Stylus Publishing, LLC

Un modelo para la autoevaluación de la calidad de programas de educación universitaria virtual. Renata Marciniak y Joaquín Gairín Sallán. 
Shelton, K., y Moore, J.C. (2011).Quality scorecard for the administration of online programs: A work in progress. Newbury Port, MA: The Sloan Consortium.

Silvio, J. (2006). Hacia una educación virtual de calidad, pero con equidad y pertinencia. RUSC: Revista de Universidad y Sociedad del Conocimiento, 3(1), 114. Recuperado de http://www.uoc.edu/rusc/3/1/dt/esp/silvio.pdf

UNIQUe. (2014). UNIQUe - European Universities Quality in e-Learning. Certifying Excellence in Institutional TEL. Recuperado de https://www.efmd.org/index.php?option=com_content\&view=article\&id=158:uniq ue-europen-university-quality-in-elearning \&catid=126:completed-projects

UW (University of Wisconsin). (2008). Logic Model. Recuperado de http://fyi.uwex.edu/programdevelopment/logic-models/

Veytia Bucheli, M. G. y Chao González, M. M. (2013). Las competencias como eje rector de la calidad educativa. Revista electrónica de Divulgación de la Investigación, 4. Recuperado de http://portales.sabes.edu.mx/redi/4/pdf/SABES_4_2MARIAPDF_V1.pdf

Zapata-Ros, M. (2015). Calidad en enseñanza abierta online universitaria: del aula virtual al MOOC. Campus Virtuales, 4(2), 86-107. Recuperado de https://issuu.com/revistacampusvirtuales/docs/7

Zapata-Ros, M. (2006). Distintas formas de intervenir en la distancia y en el eLearning Los modelos de calidad. RED. Revista de Educación a Distancia, núm. 16, 1-5. Recuperado de http://revistas.um.es/red/article/view/24231/23571

Zhang, W. y Jiang, L. (2012). Quality Assurance in E-Learning: PDPP Evaluation Model and its Application. The International Review of Research in Open and Distance Learning, 13(3), 66-82. Recuperado de http://www.irrodl.org/index.php/irrodl/article/view/1181

\section{Anexo 1}

Sistema de indicadores para el modelo de autoevaluación de programas de educación universitaria virtual.

\begin{tabular}{|l|l|}
\hline \multicolumn{1}{|c|}{ Código } & \multicolumn{1}{c|}{ Denominación } \\
\hline Dimensión 1: Justificación del programa virtual \\
\hline APEV-1 & Respuesta del programa virtual a las necesidades formativas de la sociedad. \\
\hline APEV-2 & Pertinencia social del programa virtual. \\
\hline APEV-3 & $\begin{array}{l}\text { Importancia del programa virtual para el aprendizaje y desarrollo profesional del } \\
\text { estudiante. }\end{array}$ \\
\hline Dimensión 2: Objetivos formativos del programa virtual \\
\hline APEV-4 & $\begin{array}{l}\text { Adecuación de los objetivos formativos del programa virtual a la demanda de especialistas } \\
\text { del mercado laboral. }\end{array}$ \\
\hline APEV-5 & Redacción de los objetivos formativos en función de competencias. \\
\hline APEV-6 & Coherencia de los objetivos formativos con el temario del programa virtual. \\
\hline APEV-7 & Evaluabilidad de los objetivos formativos del programa virtual. \\
\hline APEV-8 & Disponibilidad de los objetivos formativos del programa virtual. \\
\hline Dimensión 3: Perfil del estudiante \\
\hline Subdimensión 3.1: Perfil de ingreso \\
\hline APEV-9 & Establecimiento del perfil de los destinatarios del programa virtual. \\
\hline
\end{tabular}

Un modelo para la autoevaluación de la calidad de programas de educación universitaria virtual. Renata Marciniak y Joaquín Gairín Sallán. 


\begin{tabular}{|c|c|}
\hline APEV-10 & Requisitos de formación previa del aspirante al programa virtual. \\
\hline APEV-11 & Disponibilidad del perfil de ingreso del programa virtual. \\
\hline \multicolumn{2}{|c|}{ Subdimensión 3.2: Perfil de egreso } \\
\hline APEV-12 & Definición del perfil de egreso del programa virtual. \\
\hline APEV-13 & Declaración del perfil de egreso en función de competencias. \\
\hline APEV-14 & Actualización del perfil de egreso del programa virtual. \\
\hline APEV-15 & Disponibilidad del perfil de egreso del programa virtual. \\
\hline \multicolumn{2}{|c|}{ Dimensión 4: Contenido temático/temario del programa virtual } \\
\hline APEV-16 & Adecuación del contenido temático del programa a la asignatura. \\
\hline APEV-17 & Adecuación del contenido temático a la carga lectiva del programa virtual. \\
\hline APEV-18 & Coherencia entre el contenido temático y los objetivos formativos del programa virtual. \\
\hline APEV-19 & Ordenación lógica de los temas y subtemas del contenido temático del programa virtual. \\
\hline APEV-20 & Actualización del contenido temático de programa virtual. \\
\hline \multicolumn{2}{|c|}{ Dimensión 5: Actividades de aprendizaje } \\
\hline APEV-21 & Variedad de las actividades de aprendizaje del programa virtual. \\
\hline APEV-22 & $\begin{array}{l}\text { Coherencia entre las actividades de aprendizaje y los objetivos formativos del programa } \\
\text { virtual. }\end{array}$ \\
\hline APEV-23 & Directrices para realizar las actividades de aprendizaje. \\
\hline APEV-24 & Cronograma de las actividades de aprendizaje. \\
\hline APEV-25 & Fecha de entrega de las actividades. \\
\hline APEV-26 & Método de entrega de las actividades de aprendizaje. \\
\hline APEV-27 & Promoción del aprendizaje colaborativo a través de las actividades. \\
\hline APEV-28 & $\begin{array}{l}\text { Adecuación de las actividades de aprendizaje a la carga lectiva del programa virtual y a la } \\
\text { carga docente/ estudiante. }\end{array}$ \\
\hline \multicolumn{2}{|c|}{ Dimensión 6: Perfil del docente en línea } \\
\hline APEV-29 & Adecuación del perfil profesional del docente en línea en cuanto a la asignatura impartida. \\
\hline APEV-30 & Competencias pedagógicas del docente en línea. \\
\hline APEV-31 & Competencias tecnológicas del docente en línea. \\
\hline \multicolumn{2}{|c|}{ Dimensión 7: Materiales y recursos didácticos } \\
\hline \multicolumn{2}{|c|}{ Subdimensión 7.1: Unidad Didáctica } \\
\hline APEV-32 & Referencia el título de la Unidad Didáctica a su contenido. \\
\hline APEV-33 & Claridad del título de la Unidad Didáctica. \\
\hline APEV-34 & Carácter exhaustivo del índice de la Unidad Didáctica \\
\hline APEV-35 & Indicación de las páginas de cada tema en el índice. \\
\hline APEV-36 & Presentación de la introducción a la Unidad Didáctica. \\
\hline APEV-37 & Resumen del contenido de la Unidad Didáctica en la introducción. \\
\hline APEV-38 & Función motivadora de la introducción a la Unidad Didáctica. \\
\hline APEV-39 & Presentación de los objetivos formativos de la Unidad Didáctica. \\
\hline APEV-40 & Adecuación de objetivos de la Unidad Didáctica al perfil del estudiante. \\
\hline APEV-41 & Actualización de la información presentada en los contenidos de la Unidad Didáctica. \\
\hline APEV-42 & $\begin{array}{l}\text { Coherencia de contenidos de la Unidad Didáctica con los objetivos formativos de la } \\
\text { misma. }\end{array}$ \\
\hline APEV-43 & Profundización de los contenidos de la Unidad Didáctica. \\
\hline APEV-44 & Recursos multimedia de los contenidos de la Unidad Didáctica. \\
\hline APEV-45 & Atracción de los contenidos de la Unidad Didáctica. \\
\hline APEV-46 & Cumplimento de los derechos de autor. \\
\hline APEV-47 & Presentación del cierre de la Unidad Didáctica. \\
\hline APEV-48 & Presentación de la bibliografía de la Unidad Didáctica. \\
\hline APEV-49 & Presentación de la bibliografía complementaria de la Unidad Didáctica. \\
\hline APEV-50 & Actualización de bibliografía de la Unidad Didáctica. \\
\hline APEV-51 & Presentación de glosario de la Unidad Didáctica. \\
\hline APEV-52 & Existencia de ejercicios de actividades. \\
\hline APEV-53 & Ejercicios de autoevaluación de aprendizaje. \\
\hline APEV-54 & Existencia de soluciones de ejercicios de autoevaluación de aprendizaje \\
\hline \multicolumn{2}{|c|}{ Subdimensión 7.2: Guía Didáctica } \\
\hline APEV-55 & Identificación del programa virtual. \\
\hline
\end{tabular}

Un modelo para la autoevaluación de la calidad de programas de educación universitaria virtual. Renata Marciniak y Joaquín Gairín Sallán. 


\begin{tabular}{|c|c|}
\hline APEV-56 & Î́ndice de la Guía Didáctica. \\
\hline APEV-57 & Presentación del docente en línea. \\
\hline APEV-58 & Introducción al programa virtual. \\
\hline APEV-59 & Presentación de los objetivos y las competencias del programa virtual. \\
\hline APEV-60 & Presentación de contenidos temáticos del programa virtual. \\
\hline APEV-61 & Metodología de enseñanza-aprendizaje. \\
\hline APEV-62 & Actividades de aprendizaje. \\
\hline APEV-63 & Evaluación de aprendizaje. \\
\hline APEV-64 & Bibliografía del programa. \\
\hline APEV-65 & Tutoría. \\
\hline \multicolumn{2}{|c|}{ Subdimensión 7.3: Otros materiales y recursos didácticos } \\
\hline APEV-66 & Materiales y recursos didácticos complementarios. \\
\hline APEV-67 & Recursos didácticos basados en web. \\
\hline APEV-68 & Criterios de selección de los materiales y recursos didácticos del programa virtual. \\
\hline APEV-69 & Validez de los materiales y recursos didácticos del programa virtual. \\
\hline \multicolumn{2}{|c|}{ Dimensión 8: Estrategias didácticas } \\
\hline APEV-70 & Variedad de estrategias de enseñanza utilizadas por el docente en línea. \\
\hline APEV-71 & $\begin{array}{l}\text { Coherencia de las estrategias de enseñanza con los objetivos formativos del programa } \\
\text { virtual. }\end{array}$ \\
\hline APEV-72 & Promoción de estrategias de aprendizaje entre los estudiantes. \\
\hline \multicolumn{2}{|c|}{ Dimensión 9: Tutoría } \\
\hline APEV-73 & $\begin{array}{l}\text { Información sobre las funciones del docente en línea y otras personas involucradas en el } \\
\text { desarrollo del programa virtual. }\end{array}$ \\
\hline APEV-74 & Instrucciones sobre la forma de comunicación con el docente en línea. \\
\hline APEV-75 & Presentación del horario de las tutorías realizadas por el docente en línea. \\
\hline APEV-76 & Realización de tutorías individuales por el docente en línea. \\
\hline APEV-77 & Realización de tutorías grupales por el docente en línea. \\
\hline APEV-78 & Seguimiento de tutorías realizadas por el docente en línea. \\
\hline APEV-79 & Retroalimentación del docente en línea sobre el aprendizaje. \\
\hline \multicolumn{2}{|c|}{ Dimensión 10: Evaluación del aprendizaje de los estudiantes } \\
\hline APEV-80 & Estrategias de evaluación de aprendizaje. \\
\hline APEV-81 & Claridad y acceso a los criterios de evaluación de aprendizaje. \\
\hline APEV-82 & Claridad y acceso a los criterios de calificación. \\
\hline APEV-83 & Involucramiento de los estudiantes en el proceso de evaluación de aprendizaje. \\
\hline \multicolumn{2}{|c|}{ Dimensión 11: Calidad del aula virtual del programa } \\
\hline APEV-84 & Existencia de un espacio para enviar tareas o ficheros. \\
\hline APEV-85 & Existencia de herramientas de gestión de actividades. \\
\hline APEV-86 & Existencia de espacios de trabajo en grupos. \\
\hline APEV-87 & Existencia de herramientas para crear un PLE. \\
\hline APEV-88 & Existencia de herramientas de comunicación asincrónica. \\
\hline APEV-89 & Existencia de herramientas de comunicación sincrónica. \\
\hline APEV-90 & Posibilidad de seguimiento del progreso por el propio estudiante. \\
\hline APEV-91 & Posibilidad de seguimiento del progreso de los estudiantes por partes ispidel docente en línea. \\
\hline APEV-92 & Descripción de las herramientas disponibles en el aula virtual. \\
\hline \multicolumn{2}{|c|}{ Dimensión 12: Evaluación de la fase inicial del programa virtual } \\
\hline APEV-93 & Preparación del programa virtual con antelación. \\
\hline APEV-94 & Disponibilidad de los recursos. \\
\hline APEV-95 & Preparación de las personas involucradas en el programa virtual. \\
\hline APEV-96 & Preparación del aula virtual. \\
\hline \multicolumn{2}{|c|}{ Dimensión 13: Evaluación de la fase de desarrollo del programa virtual } \\
\hline APEV-97 & Adecuación de las estrategias de enseñanza para el logro de los objetivos formativos. \\
\hline APEV-98 & $\begin{array}{l}\text { Adecuación de las actividades de aprendizaje para la adquisición de las competencias } \\
\text { planteadas. }\end{array}$ \\
\hline APEV-99 & Nivel de cumplimiento del cronograma del programa virtual. \\
\hline APEV-100 & Involucramiento del docente en línea en la realización del programa virtual. \\
\hline APEV-101 & Adecuación de los materiales y recursos didácticos a las expectativas de los estudiantes. \\
\hline
\end{tabular}

Un modelo para la autoevaluación de la calidad de programas de educación universitaria virtual. Renata Marciniak y Joaquín Gairín Sallán.

Página 29 de 30 


\begin{tabular}{|l|l|}
\hline APEV-102 & Nivel de motivación de los estudiantes para el aprendizaje. \\
\hline APEV-103 & Nivel de consecución de algunos objetivos formativos del programa. \\
\hline Dimensión 14: Evaluación de la fase final del programa virtual \\
\hline APEV-104 & Nivel de consecución de los objetivos formativos del programa. \\
\hline APEV-105 & Nivel de cumplimento de las actividades de aprendizaje programadas. \\
\hline APEV-106 & Nivel de cumplimiento de sus funciones por el docente en línea. \\
\hline APEV-107 & Nivel de satisfacción de los estudiantes con el programa. \\
\hline APEV-108 & Tasa de impacto del programa virtual. \\
\hline APEV-109 & Tasa de rendimiento del programa virtual. \\
\hline APEV-110 & Tasa de éxito del programa virtual. \\
\hline APEV-111 & Tasa de no presentados. \\
\hline
\end{tabular}

Un modelo para la autoevaluación de la calidad de programas de educación universitaria virtual. 